\title{
MODELO DE PLANEJAMENTO DE ALOCAÇÃO DE PESSOAL EM SERVIÇOS DE TREINAMENTO EM TI
}

\section{PERSONNEL SCHEDULING MODEL FOR IT TRAINNING SERVICES}

\author{
Rodolfo Cunha Oliveira ${ }^{1}$; Matheus Pereira Garuti Mauricio ${ }^{2}$; Luis Antonio De Santa-Eulalia ${ }^{3}$ \\ Antonio Carlos Moretti ${ }^{4}$ \\ ${ }^{1}$ Universidade Estadual de Campinas (UNICAMP) - Faculdade de Ciências Aplicadas - Brasil \\ cunha.rodolfo@gmail.com \\ ${ }^{2}$ Universidade Estadual de Campinas (UNICAMP) - Faculdade de Ciências Aplicadas - Brasil \\ matheusgaruti@gmail.com \\ ${ }^{3}$ Université de Sherbrooke - Faculté d'administration - Canadá \\ 1.santa-eulalia@usherbrooke.ca \\ ${ }^{4}$ Universidade Estadual de Campinas (UNICAMP) - Faculdade de Ciências Aplicadas - Brasil \\ antonio.moretti@fca.unicamp.br
}

\begin{abstract}
Resumo
Empresas de Tecnologia de Informação (TI) normalmente incluem a capacitação de usuários nos seus serviços prestados. Devido a forte concorrência mercadológica, o treinamento pode ser considerado um elemento importante no portfólio de serviços oferecidos, tendo impacto nas vendas e na fidelização de clientes. No entanto, a programação e alocação de treinadores aos cursos ministrados pode ser uma tarefa deveras complexa, principalmente quando se visa a otimização dos serviços oferecidos com custos bem gerenciados, evitando desperdícios com subcontratações ou ociosidade da força de trabalho. A literatura cientifica carece de trabalhos com foco em serviços de treinamentos em TI, com periodos acíclicos e anuais de replanejamento. Além do mais, poucos são os estudos que apresentam abordagens de simples implementação e utilização, viabilizando o uso nas empresas. Dessa forma, o presente estudo propõe uma modelagem matemática do Problema de Alocação de Pessoal, juntamente com um estudo de caso visando à validação preliminar do modelo junto a uma empresa multinacional. A empresa pode facilmente identificar a necessidade de contratação, de modo evitar sobrecarga ou subcontratações, garantindo a qualidade do serviço. Conclui-se o estudo apontando futuras melhorias a serem implementadas ao modelo e possiveis caminhos a serem seguidos em próximas pesquisas.
\end{abstract}

Palavras-chave: planejamento alocação de pessoal; programação linear inteira; empresa de tecnologia de informação; estudo de caso.

\section{Introdução}

Atualmente, o mercado de consultoria e treinamento tem se mostrado cada vez mais dinâmico, acompanhando o passo acelerado dos avanços tecnológicos. As empresas de tecnologia que antes se preocupavam apenas com os planejamentos minuciosos para as linhas de produção e com as equipes de desenvolvimento, agora se deparam com um novo problema: o serviço de treinamento do usuário. 
Isto se dá, pois, devido à velocidade com que as tecnologias novas surgem, algumas empresas necessitam treinar o usuário para que este se acostume com o equipamento ou software novo com o objetivo de satisfazer a necessidade do cliente, que está cada vez mais exigente. Aliado a isso, o elevado grau de competitividade entre as empresas, principalmente na área de tecnologia, estimula as companhias a fornecerem serviços diferenciados e que agradem o cliente no período de pós-venda, com o objetivo de tornar o consumidor fiel à empresa.

O serviço de consultoria ao cliente seja ele efetuado pela empresa fornecedora de tecnologia, ou por empresas voltadas especificamente para o ramo de consultoria, muitas vezes demanda um planejamento minucioso e detalhado, sendo que este pode representar um elevado grau de complexidade devido ao número de restrições do problema, envolvendo limitações de cada treinador na equipe, de disponibilidade do cliente e até de disponibilidade de funcionários. Pode-se ainda considerar as dificuldades humanas, em que diversas leis trabalhistas devem ser respeitadas, além de a moral dos treinadores ser determinante para passar uma boa impressão para empresas cliente.

Além da dificuldade em identificar e considerar as restrições do planejamento, muitas vezes é importante diminuir o custo total do planejamento em si, seja ao se tentar minimizar a quantidade de treinamentos não atendidos, o que implicaria em uma perda de confiança, ou a quantidade de subcontratações recorrentes realizadas, ambos os casos de possíveis agendamentos ineficientes que acarretariam em um maior encargo a empresa prestadora do serviço.

Dessa forma, diversos pesquisadores (e.g. Van Den Bergh, 2013) tentam endereçar tal tipo de problema, sendo uma das abordagens utilizadas a aplicação de métodos matemáticos de Pesquisa Operacional para dimensionar e resolver o problema considerado, conhecido por Problema de Alocação de Pessoal (Personnel Scheduling, no inglês). Utilizando a ferramenta da modelagem matemática, é possível aplicar as restrições práticas encontradas na criação de um planejamento real, de modo que a solução encontrada possa ser traduzida em um bom agendamento das atividades da empresa (ERNST, 2004; VALOUXIS et al, 2012; VAN DEN BERGH, 2013). Apesar de diversos avanços importantes na área, existe uma carência na literatura científica para problemas de alocação não periódica, acíclica, para um horizonte de um ano aplicado no setor de serviços de treinamento em tecnologia. Além do mais, tal lacuna se torna ainda maior quando se trata de modelos de complexidade aceitável no meio empresarial, ou seja, cuja implementação e gestão do modelo sejam simples para uma empresa que deseja utilizá-lo em suas atividades rotineiras, sem grandes transformações organizacionais.

Motivado por esta questão atual, o objetivo principal deste trabalho é propor um modelo matemático eficiente e de fácil compreensão para empresas de treinamento de médio porte (em torno de 50 treinadores), capaz de efetuar o planejamento dos treinadores que ministram cursos 
semanais em um período anual. Tal modelo foi implementado para atender a necessidade da empresa Motorola Solutions Ltda., em que foi observada (pelo gerente do setor de treinamentos) a necessidade de apresentar uma solução inteligente para se gerenciar a complexa programação de pessoal e treinamentos. O caso da Motorola permitiu que o modelo fosse pré-testado em um problema real, verificando-se seus benefícios.

Sendo assim, o presente manuscrito está organizado da seguinte forma. Na Seção 2 apresenta-se um retrospecto da pesquisa já desenvolvida para solucionar o problema mencionado. Em seguida, a Seção 3 discute a metodologia utilizada no projeto. Na Seção 4 o modelo proposto é apresentado, sendo que na Seção 5 a implementação na empresa Motorola Solutions, bem como os resultados obtidos, são discutidos. Finalmente, na Seção 6 fazem-se algumas considerações finais e discute-se possíveis trabalhos futuros na área.

\section{Revisão da literatura}

Desde a década de 50 com a introdução do Problema de Alocação de Pessoal (Personnel Scheduling) no artigo de alocação de trabalhadores em cabines de pedágio apresentado por Edie (1954) e comentado por Dantzig (1954), uma grande quantidade de artigos e relatórios abordando as mais complexas variações do problema e métodos de solução foram publicados em revistas científicas.

Uma das primeiras classificações para o problema foi apresentada por Baker (1978), na qual ele divide em três grupos básicos, de acordo com as características do mesmo. A primeira é o Planejamento de Turnos (Shift Scheduling), que tem como característica o planejamento no horizonte diário do pessoal e a alocação dos trabalhadores de acordo com os requerimentos dos turnos, podendo ocorrer sobreposição dos mesmos ou não. A segunda classificação é o Planejamento das Folgas Semanais (Days Off Scheduling), onde o número de dias de serviço é diferente do número de dias de operação da empresa, ou seja, é necessário planejar os dias de folga dos trabalhadores. Finalmente, o terceiro é o Planejamento de Ciclos (Tour Scheduling), planejamento resultante da combinação dos dois tipos anteriores, com empresas trabalhando em certos dias da semana com múltiplos turnos.

Diversas outras classificações são também atribuídas de acordo com o método utilizado para solucionar o problema. Ernst et al (2004) propõe a subdivisão em cinco categorias diferentes: modelagem da demanda (Demand Modelling), abordagens utilizando inteligência artificial (Artificial Intelligence Approaches), programação com restrições (Constraint Programming), metaheurísticas (Metaheuristics), e abordagens utilizando programação matemática (Mathematical Programming Approaches). Nesse sentido, a nossa proposta se encaixa na última classificação. 
As principais áreas de aplicações para o problema citado identificadas por Van Den Bergh et al (2013) são: serviços, transportes, manufatura, varejo, militar e geral. Na categoria geral são consideradas aplicações menos frequentes como restaurantes, supermercados, festivais, estacionamentos, entre outros.

Atualmente, as empresas buscam a formação de equipes cada vez mais reduzidas, de forma cortar custos desnecessários decorrentes de ociosidade de sua mão de obra, além do atendimento das novas necessidades dos trabalhadores de hoje em dia. Nesse sentido o Problema de Alocação de Pessoal ganhou a atenção de muitos pesquisadores nas últimas décadas.

No setor de serviços, a maioria de trabalhos publicados trata do problema de Alocação de Enfermeiros (Nurse Rostering), o qual tem como objetivo encontrar a alocação ótima de equipes de enfermeiros por turnos em um intervalo de tempo especificado. A popularidade de tal tópico culminou mesmo em uma competição internacional entre pesquisadores, a International Nurse Rostering Competition, promovida pela conferência líder no assunto, the International Conference on the Practice and Theory of Automated Timetabling. Alguns dos artigos mais recentes publicados (VALOUXIS, 2012; GLASS e KNIGHT, 2010; BURKE et al 2010), apresentam métodos de Programação Linear Inteira para encontrar soluções para o problema.

Já em relação ao setor de transportes, o foco dos estudos é relacionado ao problema de Programação de Tripulação (Crew Scheduling), tendo suas principais aplicações diretas em empresas aéreas e de transporte coletivo, abordando o conceito de esquematizar as equipes de bordo nos voos, os motoristas de ônibus e os operadores de trens. Artigos como os de Saddoune et al (2011) e de Jütte e Thonemann (2012), aplicam o método de geração de colunas e otimização combinatória para melhorar a qualidade das escalas feitas por linhas aéreas e ferroviárias, minimizando o custo das empresas nesse processo.

Da mesma maneira, artigos como Kabak et al (2008), Zhang et al (2012), Chong e Strevell (1985), demonstram diferentes abordagens de Problemas de Alocação de Pessoal nos setores de varejo, manufatura e militar, respectivamente. Além disso, diversos autores tratam de Problemas de Alocação de Pessoal em escolas ou universidades, porém, foi encontrado somente um artigo (HAASE et al, 1998) que possui o problema formulado com características básicas parecidas com o proposto nesse artigo.

$\mathrm{O}$ artigo mencionado apresenta o problema da Lufthansa Technical Training GmbH, em que a empresa oferece 670 diferentes tipos de cursos de treinamento, dos quais centenas são ministrados anualmente. O objetivo do artigo é solucionar o problema de planejamento dos cursos de modo criar uma agenda anual que maximize a margem de lucro da empresa, atendendo a diferentes tipos de restrições. A principal semelhança é o fato de se estar, no presente trabalho, propondo uma alocação não periódica para um horizonte de um ano. Pelo fato de este tópico ser pouco explorado, parte-se 
do trabalho citado como ponto de partida, visando propor um modelo de fácil compreensão por parte da empresa e de simples implementação, visando aumentar sua aceitação. No entanto, tal modelagem pode ser futuramente sofisticada de acordo com as necessidades das empresas de treinamento.

\section{Metodologia}

Este estudo alia aspectos de pesquisa instrumental com a pesquisa exploratória e estudo de caso. Esses três aspectos são discutidos a seguir:

- Pesquisa instrumental: antes de nada, a proposta de um modelo de otimização com enfoque aplicado faz uso das premissas básicas da pesquisa dita “instrumental”, a qual é recomendada para o campo de pesquisas aplicadas (MARTEL, 1986; MATTESSICH, 1978).

(...) refere-se às atividades criativas metodológicas que visam a criação de um instrumento visando dar suporte às organizações na realização de suas finalidades. Esses instrumentos incluem sistemas, métodos, modelos, bem como componentes desses instrumentos, e as ferramentas necessárias para inventá-los.

No presente caso, o instrumento proposto refere-se a um novo modelo de Personnel Schedulling para empresa de tecnologia. Na expectativa de produzir tal instrumento, utiliza-se basicamente de métodos de síntese e de análise. Normalmente, a pesquisa instrumental faz uso de uma fase dita criativa (chamada de "inteligência" por Martel (1986)), na qual o instrumento é desenvolvido. Após tal etapa, dá-se sequência a outra visando a validação do instrumento criado. Assim, neste trabalho, alguns testes do modelo proposto foram realizados. No entanto, como a validação foi apenas preliminar e não realizada de forma extensiva, i.e. em diversas situações distintas que permitem uma eventual generalização, este trabalho também apresenta características de pesquisa exploratória;

- Pesquisa exploratória: esse tipo de pesquisa tem como objetivo “(...) proporcionar maior familiaridade com o problema com vistas a torná-lo explícito ou a construir hipóteses”. Pode envolver, entre outras coisas, análise de exemplos que estimulem a compreensão e pode assumir, em diversas situações, a forma de "estudos de caso" (SILVA e MENESES, 2005, p. 21). Os testes realizados neste trabalho levantam hipóteses quanto à utilidade e facilidade de uso do instrumento proposto, abrindo portas para estudos mais aprofundados no futuro.

- Estudo de caso: o estudo de caso (YIN, 2009) visa fornecer condições para uma validação preliminar do modelo proposto. Para tanto, uma aplicação real em escala industrial foi realizada junto à empresa Motorola Solutions Ltda.

Na próxima seção, o instrumento proposto será apresentado. 


\section{Modelo proposto}

A fim de resolver o problema mencionado acima, foi proposto, a partir de formulações como as apresentadas por Daskalaki et al (2004) e Al-Yakoob e Sherali (2006), um modelo matemático de Programação Linear Inteira (PLI), tendo como principal objetivo a minimização do número de funcionários empregados e subcontratados para atender a demanda total de treinamentos durante o ano. A seguir estão as informações sobre os parâmetros, índices, variáveis e conjuntos utilizados no modelo.

\section{Índices e parâmetros}

$N$ : Número de treinadores;

$P$; Quantidade de tipos de treinamento;

T: Período total;

$i=1,2, \ldots, N$ : i-ésimo treinador;

$k=1,2, \ldots, P: \mathrm{k}$-ésimo tipo de treinamento;

$t=1,2, \ldots, T:$ t-ésimo período;

$d_{t k}:$ demanda do treinamento $k$ no período $t$

$M$ : número inteiro arbitrário e grande.

\section{Variáveis}

$x_{i t k}$ : variável binária que é 1 se o treinador $i$, ministrou o treinamento do tipo $k$, no período $t$

$z_{i \bar{t} t}$ : variável binária que é 1 se o treinador $i$, ministrou algum treinamento no período $t ;$

$y_{t k}:$ variável inteira que representa quantas subcontratações ocorreu para o treinamento do tipo $k$, no período $t$.

\section{Conjuntos}

$I(k)$ : Conjunto de subconjuntos que representam o índice dos treinadores que podem ministrar o treinamento do tipo $k$.

Assim, tem-se o modelo em PLI (Programação Linear Inteira), que é uma classe de problemas que tem como característica a facilidade de implementação e obtenção de resultados para exemplos de pequeno porte como o da empresa considerada nesse estudo. A grande questão dessa classe de problemas é a imprevisibilidade resultante com o possível aumento de magnitude dos índices considerados, conforme o apresentado nas seções a seguir. O modelo então é formulado da seguinte forma: 


\section{Modelo matemático}

$$
\operatorname{Minimizar} \sum_{t}\left(\sum_{i} z_{i t}+\sum_{k} M y_{c k}\right)
$$

$$
\begin{aligned}
& \text { Sujeito à } \\
& \sum_{i \in r(k)}\left(x_{i t k}\right)+y_{t k} \geq d_{t k} \\
& \forall t=1,2 \ldots, T ; \forall k=1,2 \ldots P \\
& z_{i t}=\sum_{k}\left(x_{i t k}\right), \\
& \forall i=1,2 \ldots, N ; \forall t=1,2 \ldots T \\
& z_{i t}+z_{i t+1}+z_{i t+2} \leq 2 \text {, } \\
& \forall i=1,2 \ldots, N ; \forall t=1,2 \ldots T \\
& y_{t k} \in \mathbb{N}_{;} \quad x_{i t k}, z_{i t} \in\{0,1\}
\end{aligned}
$$

A Função objetivo (1) busca minimizar a quantidade de funcionários alocados, para não haver alocações desnecessárias, e penaliza caso alguma subcontratação seja feita, deste modo, a subcontratação só ocorrerá no caso de infactibilidade, podendo ser representada pela falta de funcionários disponíveis ou por um excesso na demanda.

As restrições do tipo (2) têm como função forçar que a quantidade de funcionários alocados ou subcontratados no período $t$ seja suficiente para satisfazer a demanda daquele período, para todo $k$. É importante ressaltar que o somatório em $i$ contém apenas os treinadores que podem ministrar o treinamento do tipo $k$.

Em (3), garante-se que a variável $z_{i t}$ esteja ativa caso o treinador $l$ tenha ministrado algum treinamento no período $t$.

As restrições (4) forçam que o treinador $i$ não trabalhe mais de dois períodos consecutivamente.

Finalmente, o bloco de restrições (5) garante a integralidade das variáveis $y_{t k}$ e que as variáveis $x_{i t k}$ e $z_{i t}$ sejam binárias.

O modelo proposto foi resolvido pelo software livre de otimização linear GLPK, versão 4.49, utilizando um computador com 8GB de memória RAM e um processador i5-3570k de 3.4 $\mathrm{GHz}$.

\section{Caso Motorola Solutions LTDA}

\subsection{A empresa}


A multinacional Motorola Solutions é fruto da segregação ocorrida na Motorola e é vista por muitos como a sucessora direta da antiga empresa fundada em 1928. Conhecida pela sua potente infraestrutura e alta capacidade de inovação, a Motorola encontra-se no Brasil desde 1994 onde instaurou uma fábrica em Jaguariúna, no interior do Estado de São Paulo e escritório em São Paulo, capital. Após a separação ocorrida em 2011, os escritórios da Motorola Solutions no Brasil foram transferidos para Barueri, em São Paulo.

Atualmente a Motorola Solutions é responsável pelo mercado de dados de comunicação e equipamentos de telecomunicação. A estrutura da empresa possui duas divisões, a empresarial e a governamental. A divisão empresarial desenvolve soluções para captura avançada de dados, infraestrutura sem fio, leitura de códigos de barra, rádios bidirecionais e pagers de negócios, redes de banda larga sem fio e soluções de RFID para clientes no mundo inteiro. Por outro lado, a divisão governamental produz segurança pública e produtos diversos para o governo, incluindo rádios bidirecionais analógicos e digitais, produtos de comunicações de voz e dados, sistemas de segurança para redes sem fio, computação móvel, entre outros.

Os produtos oferecidos e desenvolvidos pela empresa estão vinculados aos treinamentos de capacitação para o usuário. Os treinamentos são realizados semanalmente e ocorrem em praticamente toda a América Latina durante o ano inteiro, tendo suas datas estipuladas pelo contratante, permitindo-se pouca ou nenhuma flexibilidade.

O número de treinadores fornecidos pela Motorola Solutions é fixo e cada um deles é capaz de dar tipos diferentes de treinamentos. Devido à necessidade de evitar sobrecargas para os treinadores, é exigido que um treinador não trabalhasse mais do que duas semanas seguidas, isso por que os cursos são ministrados em outras cidades, exigindo que ocorra uma locomoção, muitas vezes aérea, até a empresa cliente. A subcontratação de treinadores é realizada com frequência.

\subsection{Obtenção de dados}

Para validar e avaliar o modelo, foram utilizados os dados fornecidos pelo gerente da equipe de treinadores da América Latina da empresa Motorola Solutions referentes aos treinamentos já realizados e que ainda seriam realizados no ano de 2013. Além disso, visando testar a eficiência do modelo, foram gerados aleatoriamente cenários variando o número de treinadores $N$ e os tipos de treinamentos existentes $P$. A obtenção desses cenários visou testar o modelo em situações de eventual crescimento da demanda, devido, entre outras coisas, a grandes eventos esportivos que se realização no Brasil em 2014 (Copa do Mundo de Futebol) e 2016 (Jogos Olímpicos). 


\section{Resultados}

Tal como mencionado anteriormente, o problema foi resolvido pelo solver GLPK para diferentes cenários, incluindo o cenário real proposto pela empresa analisada e cenários aleatórios gerados. Esta seção apresenta os dados e a solução encontrada para o problema real e demonstra apenas os tempos computacionais e os resultados obtidos para cada cenário.

A Tabela 1 contém as informações básicas a todos cenários:

Tabela 1 - Dados comuns aos cenários

\begin{tabular}{|c|c|c|c|}
\hline Cenário & Número de treinadores $(\mathrm{N})$ & $\begin{array}{l}\text { Tipos de treinamento } \\
\text { (P) }\end{array}$ & Total de Períodos (T) \\
\hline Motorola Solutions & 4 & 6 & 45 \\
\hline Caso 1 & 5 & 10 & 45 \\
\hline Caso 2 & 10 & 20 & 45 \\
\hline Caso 3 & 15 & 40 & 45 \\
\hline Caso 4 & 20 & 50 & 45 \\
\hline Caso 5 & 25 & 70 & 45 \\
\hline Caso 6 & 30 & 85 & 45 \\
\hline Caso 7 & 35 & 90 & 45 \\
\hline Caso 8 & 40 & 100 & 45 \\
\hline Caso 9 & 45 & 130 & 45 \\
\hline Caso 10 & 50 & 150 & 45 \\
\hline
\end{tabular}

Fonte: autoria própria (2014)

É válido ressaltar também, que o valor do inteiro grande $M$ mencionado na modelagem, foi de 10000 para todos os cenários.

Pode-se, então, focar no caso proposto pela empresa, sendo que as Tabelas 2 e 3 apresentam a demanda real para cada tipo de treinamento para um período de 45 semanas ( 22 semanas na Tabela 2 e 23 semanas na Tabela 3 ).

Tabela 2 - Demanda a ser planejada das 22 primeiras semanas

\begin{tabular}{cccccccccccccccccccccccc}
\hline Tipo & $\mathbf{1}$ & $\mathbf{2}$ & $\mathbf{3}$ & $\mathbf{4}$ & $\mathbf{5}$ & $\mathbf{6}$ & $\mathbf{7}$ & $\mathbf{8}$ & $\mathbf{9}$ & $\mathbf{1 0}$ & $\mathbf{1 1}$ & $\mathbf{1 2}$ & $\mathbf{1 3}$ & $\mathbf{1 4}$ & $\mathbf{1 5}$ & $\mathbf{1 6}$ & $\mathbf{1 7}$ & $\mathbf{1 8}$ & $\mathbf{1 9}$ & $\mathbf{2 0}$ & $\mathbf{2 1}$ & $\mathbf{2 2}$ \\
\hline 1 & 0 & 1 & 0 & 0 & 0 & 0 & 1 & 0 & 1 & 0 & 1 & 0 & 0 & 1 & 1 & 0 & 1 & 1 & 0 & 1 & 0 & 0 \\
2 & 1 & 0 & 0 & 0 & 0 & 0 & 0 & 0 & 1 & 2 & 0 & 1 & 0 & 0 & 0 & 2 & 0 & 0 & 2 & 0 & 0 & 0 \\
3 & 1 & 0 & 0 & 0 & 0 & 1 & 0 & 0 & 0 & 0 & 0 & 0 & 0 & 0 & 0 & 0 & 0 & 0 & 0 & 0 & 0 & 0 \\
4 & 0 & 0 & 0 & 0 & 0 & 0 & 0 & 0 & 0 & 0 & 0 & 0 & 0 & 0 & 0 & 0 & 0 & 0 & 0 & 1 & 0 & 1 \\
5 & 0 & 0 & 0 & 0 & 0 & 0 & 0 & 0 & 0 & 0 & 0 & 0 & 0 & 0 & 0 & 0 & 0 & 0 & 0 & 0 & 0 & 0 \\
6 & 0 & 0 & 0 & 0 & 0 & 0 & 0 & 0 & 0 & 0 & 0 & 0 & 0 & 0 & 0 & 0 & 0 & 0 & 0 & 0 & 0 & 0 \\
\hline
\end{tabular}

Fonte: Pesquisa de campo (2013)

Tabela 3 - Demanda a ser planejada das 23 semanas restantes

\begin{tabular}{cccccccccccccccccccccccc}
\hline \multirow{2}{*}{ Tipo } & $\mathbf{2 3}$ & $\mathbf{2 4}$ & $\mathbf{2 5}$ & $\mathbf{2 6}$ & $\mathbf{2 7}$ & $\mathbf{2 8}$ & $\mathbf{2 9}$ & $\mathbf{3 0}$ & $\mathbf{3 1}$ & $\mathbf{3 2}$ & $\mathbf{3 3}$ & $\mathbf{3 4}$ & $\mathbf{3 5}$ & $\mathbf{3 6}$ & $\mathbf{3 7}$ & $\mathbf{3 8}$ & $\mathbf{3 9}$ & $\mathbf{4 0}$ & $\mathbf{4 1}$ & $\mathbf{4 2}$ & $\mathbf{4 3}$ & $\mathbf{4 4}$ & $\mathbf{4 5}$ \\
\hline 1 & 1 & 0 & 0 & 0 & 0 & 0 & 1 & 0 & 1 & 0 & 0 & 0 & 0 & 0 & 0 & 1 & 1 & 0 & 0 & 1 & 0 & 0 & 1 \\
2 & 0 & 0 & 0 & 0 & 1 & 0 & 0 & 2 & 0 & 2 & 1 & 1 & 1 & 1 & 1 & 0 & 0 & 0 & 1 & 0 & 0 & 0 & 0 \\
3 & 0 & 0 & 0 & 0 & 0 & 0 & 0 & 0 & 0 & 0 & 0 & 0 & 0 & 0 & 0 & 0 & 0 & 0 & 0 & 0 & 0 & 0 & 0 \\
4 & 0 & 0 & 0 & 0 & 0 & 0 & 0 & 0 & 2 & 0 & 0 & 0 & 0 & 0 & 0 & 0 & 0 & 0 & 0 & 1 & 0 & 1 & 0 \\
5 & 0 & 0 & 0 & 0 & 0 & 0 & 1 & 0 & 0 & 0 & 0 & 0 & 0 & 0 & 0 & 0 & 0 & 0 & 1 & 0 & 1 & 0 & 0 \\
6 & 0 & 1 & 0 & 0 & 0 & 0 & 0 & 0 & 0 & 0 & 0 & 0 & 0 & 0 & 0 & 0 & 0 & 0 & 0 & 0 & 0 & 0 & 0 \\
\hline
\end{tabular}

Fonte: Pesquisa de campo (2013)

Os conjuntos $I(k)$ mencionados na metodologia foram definidos da seguinte maneira: 
$I(1):\{1\}$

$I(2):\{1,2\}$;

$I(3) ;\{1,2,3\}$;

$I(4):\{1,3\}$;

$I(5):\{2\} ;$

$I(6):\{1,2,3,4\}$

Assim, o problema foi resolvido através do solver GLPK e a resposta obtida é exibida por meio de diagramas de Gantt, nas Figuras 1 e 2, a seguir.

Figura 1 - Planejamento das 22 primeiras semanas

\begin{tabular}{|c|c|c|c|c|c|c|c|c|c|c|c|c|c|c|c|c|c|c|c|c|c|c|}
\hline \multirow[b]{2}{*}{ Treinador } & \multicolumn{22}{|c|}{ Semana } \\
\hline & 1 & 2 & 3 & 4 & 5 & 6 & 7 & 8 & 9 & 10 & 11 & 12 & 13 & 14 & 15 & 16 & 17 & 18 & 19 & 20 & 21 & 22 \\
\hline 1 & & & & & & & & & & & & & & & & & & & & & & \\
\hline 2 & & & & & & & & & & & & & & & & & & & & & & \\
\hline 3 & & & & & & & & & & & & & & & & & & & & & & \\
\hline 4 & & & & & & & & & & & & & & & & & & & & & & \\
\hline Sub1 & & & & & & & & & & & & & & & & & & & & & & \\
\hline Sub2 & & & & & & & & & & & & & & & & & & & & & & \\
\hline
\end{tabular}

Fonte: autoria própria (2014)

Figura 2 - Planejamento das 23 semanas restantes

\begin{tabular}{|c|c|c|c|c|c|c|c|c|c|c|c|c|c|c|c|c|c|c|c|c|c|c|c|}
\hline & \multicolumn{10}{|c|}{ Treinador } \\
\cline { 2 - 3 } & 23 & 24 & 25 & 26 & 27 & 28 & 29 & 30 & 31 & 32 & 33 & 34 & 35 & 36 & 37 & 38 & 39 & 40 & 41 & 42 & 43 & 44 & 45 \\
\hline 1 & & & & & & & & & & & & & & & & & & & & & & & \\
\hline 2 & & & & & & & & & & & & & & & & & & & & & & & \\
\hline 3 & & & & & & & & & & & & & & & & & & & & & & & \\
\hline 4 & & & & & & & & & & & & & & & & & & & & & & & \\
\hline Sub1 & & & & & & & & & & & & & & & & & & & & & & \\
\hline Sub2 & & & & & & & & & & & & & & & & & & & & & & & \\
\hline
\end{tabular}

Fonte: autoria própria (2014)

Pode-se notar que existe uma sobrecarga de dois treinadores (1 e 2), o que força o problema a recorrer às subcontratações, isso se deve ao fato dos treinadores 3 e 4 não possuírem qualificações para atender os treinamentos que possuem maior demanda, no caso, os treinamentos do tipo 1 e 2 .

Para contornar o problema podem-se notar duas soluções de imediato. A primeira seria a contratação de algum novo treinador capacitado nos treinamentos do tipo 1 e 2 , enquanto a outra solução seria focar em capacitar melhor os treinadores 3 e 4 já existentes na equipe.

Além do problema real, para testar a aplicação do modelo proposto, alguns cenários adicionais mencionados anteriormente nesta seção (vide Tabela 3) foram gerados a fim de determinar para quais tamanhos o problema apresenta um baixo tempo computacional.

Todos os cenários apresentados na Tabela 1 apresentaram um tempo de solução relativamente pequeno (no máximo 1 minuto), entretanto problemas maiores apresentaram tempo de solução superior a 1 hora, de modo que estes cenários foram descartados desta análise. A Tabela 4 reúne as principais informações encontradas de cada cenário testado. 
Tabela 4 - Resultados

\begin{tabular}{cccccc}
\hline Cenário & Tempo de solução(s) & $\begin{array}{c}\text { Variáveis } \\
\text { Utilizadas }\end{array}$ & $\begin{array}{c}\text { Restrições } \\
\text { Geradas }\end{array}$ & $\begin{array}{c}\text { Demanda } \\
\text { Total }\end{array}$ & Subcontratações \\
\hline Motorola Solutions & Inferior a 0.1 & 1530 & 1118 & 47 & 5 \\
Caso 1 & Inferior a 0.1 & 2925 & 4941 & 68 & 2 \\
Caso 2 & 0.1 & 10350 & 18881 & 170 & 0 \\
Caso 3 & 0.3 & 29475 & 73321 & 195 & 5 \\
Caso 4 & 0.3 & 48150 & 114261 & 312 & 4 \\
Caso 5 & 3.3 & 83025 & 222701 & 475 & 9 \\
Caso 6 & 82.7 & 119925 & 327766 & 505 & 2 \\
Caso 7 & 9.2 & 147375 & 367581 & 646 & 34 \\
Caso 8 & 8.2 & 186300 & 453521 & 680 & 9 \\
Caso 9 & 139.4 & 271125 & 764461 & 812 & 38 \\
Caso 10 & 16.6 & 346500 & 1016901 & 891 & 49 \\
\hline
\end{tabular}

Fonte: autoria própria (2014)

Comparando os resultados com os dados de entrada, citados na Tabela 1 nota-se que um singelo crescimento nos parâmetros iniciais (número de treinadores e tipos de treinamento), o tamanho do problema cresce de forma combinatorial e, consequentemente, o tempo de solução também aumenta na mesma proporção.

É importante notar que os casos 6 e 9 foram aqueles com o maior tempo de solução, e isto se deve provavelmente ao fato da matriz destes problemas possuir uma quantidade maior de simetrias, o que dificulta a determinação de uma solução ótima em tempo.

\section{Considerações finais}

O estudo realizado abordou, por meio de uma técnica de modelagem matemática, o Problema de Alocação de Pessoal para empresas de consultoria e treinamentos, com a elaboração de um modelo de Programação Linear Inteira com a capacidade de determinar um planejamento eficiente. O modelo foi pré-testado na empresa Motorola Solutions Ltda., sendo comprovada a sobrecarga dos atuais treinadores alocados atualmente na empresa.

As principais alternativas identificadas para solução do problema na empresa são: a contratação de um novo funcionário ou a capacitação dos treinadores atuais. A primeira encontra-se atualmente em estudo e a empresa, tendo em vista as futuras demandas que surgirão no período de eventos como a Copa do Mundo e as Olimpíadas no Brasil. A segunda, não é considerada no momento pela empresa, pois os treinadores com alto grau de ociosidade são, na verdade, funcionários de uma empresa terceirizada contratada pela Motorola para dar os treinamentos.

Levando em consideração os cenários gerados aleatoriamente, pôde-se notar que os tempos de solução são dependentes, em grande parte, da matriz de demandas gerada artificialmente (para eventuais aumentos de demanda no futuro). Para esse procedimento, usa-se uma rotina em linguagem $\mathrm{C}$, e em seguida resolve-se utilizando o GLPK. Notou-se que para casos em que existia mais de 100 treinadores e 150 tipos de treinamento diferentes, o GLPK não foi capaz de solucionar o problema, limitando o enfoque das aplicações propostas para empresas de treinamento de médio e 
pequeno porte. Além disso, vale ressaltar que os períodos considerados são em blocos semanais, uma vez que essa era a necessidade da Motorola.

Os resultados alcançados atingiram as metas esperadas pela empresa, e diversas sofisticações ao modelo podem ser feitas para torná-lo mais robusto e contemplar diferentes casos. Nesse sentido, uma sugestão para futuras pesquisas seria o melhoramento do modelo atual, por meio da adição de custos associados aos processos de contratação, subcontratação e capacitação dos treinadores. Além disso, para a resolução de casos com complexidade computacional maiores, sugere-se o estudo de heurísticas para determinação de planejamentos próximos do ótimo.

Uma das principais dificuldades encontradas no estudo foi o levantamento de artigos que possuíssem uma abordagem aplicada ao setor em questão, pois, apesar do problema de Personnel Scheduling possuir uma vasta literatura, sendo estudado em diversas áreas, não se encontrou artigos (senão o apresentado por Haase et al (1998)) para o setor de empresas de treinamento. Dessa maneira, o presente artigo busca contribuir para esta linha de pesquisa fornecendo um novo modelo na área.

\begin{abstract}
Information Technology (IT) companies typically include user training in their services. Due to strong marketing competition, this can be considered an important process, having an impact on sales and customer loyalty. However, this can be a difficult task, especially when it aims to optimize services with well-managed costs by avoiding waste or subcontracts and idle workforce. The scientific literature lacks studies focusing on IT training services with acyclic and annual replanning periods. Moreover, there are few studies that present approaches of simple implementation and use, encouraging their use in real companies. Thus, this study proposes a mathematical model for the Personnel Scheduling Problem in the IT training industry. A case study at an international company was performed aiming to validate the preliminary model. By using the proposed approach, the company was able to easily identify that it was necessary to hire more instructors, so as to avoid overloading or subcontracting, ensuring the quality of service. We concluded the study by pointing out some possible improvements for future research.
\end{abstract}

Keywords: personal scheduling; integer linear programming; information technology company; case study.

\title{
Referências
}

AL-YAKOOB, S. M.; SHERALI, H. D. Mathematical programming models and algorithms for a class-faculty assignment problem. European Journal of Operational Research, v. 173, n. 2, p. 488-507, 2006. crossref

BAKER, K. R. Workforce allocation in cyclical scheduling problems: A survey. Operational Research Quarterly, $1976,155-167$.

BURKE, E. K.; LI, J.; QU, R. A hybrid model of integer programming and variable neighbourhood search for highlyconstrained nurse rostering problems. European Journal of Operational Research, v. 203, n. 2, p. 484-493, 2010. crossref

CHONG, P. S.; STREVELL, M. W. A vacation scheduling algorithm for military flight crews: Maximizing satisfaction while maintaining military preparedness. Journal of Operations Management, v. 5, n. 2, p. 205-211, 1985. cross ref 
DANTZIG, G. A Comment on Edie’s Traffic Delay at Toll Booths. Operations Research, v. 2, p. 339-341, 1954.

DASKALAKI, S.; BIRBAS, T.; HOUSOS, E. An integer programming formulation for a case study in university timetabling. European Journal of Operational Research, v. 153, n. 1, p. 117-135, 2004. crossref

EDIE, L. C. Traffic delays at toll booths. Operations Research,v. 2, n. 2, p. 107-138, 1954.

ERNST, A. T.; JIANG, H; KRISHNAMOORTHY, M.; SIER, D. Staff scheduling and rostering: A review of applications, methods and models. European Journal of Operational Research, v. 153, n. 1, p. 3-27, 2004. crossref

ERNST, A. T.; JIANG, H.; KRISHNAMOORTHY, M.; OWENS, B.; SIER, D. An annotated bibliography of personnel scheduling and rostering. Annals of Operations Research, v. 127, n.1, p.1-4, 21-144, 2004.

GLASS, C. A.; KNIGHT, R. A. The nurse rostering problem: A critical appraisal of the problem structure. European Journal of Operational Research, v. 202, n. 2, p. 379-389, 2010. crossref

HAASE, K.; LATTEIER, J.; SCHIRMER, A. The course scheduling problem at Lufthansa Technical Training. European Journal of Operational Research, v. 110, n. 3, p. 441-456, 1998. crossref

JÜTTE, S.; THONEMANN, U. W. Divide-and-price: A decomposition algorithm for solving large railway crew scheduling problems. European Journal of Operational Research, v. 219, n. 2, p. 214-223, 2012. cross ref

MARTEL, A. La recherche instrumentale sectorielle en sciences de l'administration. In: La production des connaissances scientifiques de l'administration/The generation of scientific administrative knowledge. M. Audet, and Malouin, J.-L. Québec: Les Presses de l'Université Laval, 1986.

MATTESSICH, R. Instrumental reasoning and systems methodology, Reidel Pub. Co., 1978. crossref

SADDOUNE, M.; D.; G.; ELHALLAOUI, I.; SOUMIS, F. Integrated airline crew scheduling: A bi-dynamic constraint aggregation method using neighborhoods. European Journal of Operational Research, v. 212, n. 3, p. 445-454, 2011. crossref

VAlOUXIS, C., GOGOS, C; GOULAS, G.; ALEFRAGIS, P.; HOUSOS, E. A systematic two phase approach for the nurse rostering problem. European Journal of Operational Research, v. 219, n. 2, p. 425-433, 2012. crossref

VAN DEN BERGH, J.; BELIEN, J.; DEBRUECKER, P.; DEMEULEMEERSTER, E.; DEBOECK, L.. Personnel scheduling: A literature review. European Journal of Operational Research, v. 226, n .3, p. 367-385, 2012.

crossref

YIN, R.K. Case Study Research: Design and Methods, 4a edição, Thousands Oks: SAGE Publications, 2009.

ZHANG, R.; CHANG, P.; WU, C. A hybrid genetic algorithm for the job shop scheduling problem with practical considerations for manufacturing costs: investigations motivated by vehicle production. International Journal of Production Economics, v. 145, n. 1, p. 38-52, 2012. crossref

\section{Dados dos autores}

\section{Nome completo: Rodolfo Cunha Oliveira}

Filiação institucional: Universidade Estadual de Campinas - UNICAMP

Departamento: Faculdade de Ciências Aplicadas

Função ou cargo ocupado: Mestrando

Endereço completo para correspondência (bairro, cidade, estado, país e CEP): Limeira, SP, R.

Pedro Zaccaria, 1300 - Caixa Postal 1068, CEP 13484-350 - Limeira - São Paulo

Telefones para contato: (19) 3701-6675

e-mail: cunha.rodolfo@gmail.com 
Nome completo: Matheus Pereira Garuti Mauricio

Filiação institucional: Universidade Estadual de Campinas (UNICAMP)

Departamento: Faculdade de Ciências Aplicadas

Função ou cargo ocupado: Mestrando

Endereço completo para correspondência (bairro, cidade, estado, país e CEP): Limeira, SP, R.

Pedro Zaccaria, 1300 - Caixa Postal 1068, CEP 13484-350 - Limeira - São Paulo

Telefones para contato: (19) 3701-6675

e-mail:matheusgaruti@gmail.com

Nome completo: Luis Antonio De Santa-Eulalia

Filiação institucional: Université de Sherbrooke

Departamento: Faculté d'administration

Função ou cargo ocupado: Professor Adjunto

Endereço completo para correspondência (bairro, cidade, estado, país e CEP): bureau K1-4012, 2500, boul. de l'Université, Sherbrooke (Québec), CANADA, J1K 2R1

Telefones para contato: $+1(819) 821-8000$, ramal 65042

e-mail:1.santa-eulalia@usherbrooke.ca

Nome completo: Antonio Carlos Moretti

Filiação institucional: Universidade Estadual de Campinas (UNICAMP)

Departamento: Faculdade de Ciências Aplicadas

Função ou cargo ocupado: Professor Titular

Endereço completo para correspondência (bairro, cidade, estado, país e CEP): Limeira, SP, R.

Pedro Zaccaria, 1300 - Caixa Postal 1068, CEP 13484-350 - Limeira - São Paulo

Telefones para contato: (19) 3701-6675

e-mail: antonio.moretti@fca.unicamp.br

Submetido em: 21/03/204

Aceito em: 14/11/2014 